Analysis of distributed temperature sensing based on Raman scattering using OTDR coding and discrete Raman amplification

This article has been downloaded from IOPscience. Please scroll down to see the full text article.

2007 Meas. Sci. Technol. 183211

(http://iopscience.iop.org/0957-0233/18/10/S24)

View the table of contents for this issue, or go to the journal homepage for more

Download details:

IP Address: 128.178.23.47

The article was downloaded on 01/11/2012 at 14:03

Please note that terms and conditions apply. 


\title{
Analysis of distributed temperature sensing based on Raman scattering using OTDR coding and discrete Raman amplification
}

\author{
Gabriele Bolognini ${ }^{1}$, Jonghan Park ${ }^{2}$, Marcelo A Soto ${ }^{1}$, \\ Namkyoo Park ${ }^{2}$ and Fabrizio Di Pasquale ${ }^{1}$ \\ ${ }^{1}$ Scuola Superiore Sant'Anna, via G Moruzzi 1, 56124 Pisa, Italy \\ ${ }^{2}$ School of EECS, Seoul National University, Seoul, Korea \\ E-mail: gabriele.bolognini@cnit.it and nkpark@plaza.snu.ac.kr
}

Received 19 December 2006, in final form 13 February 2007

Published 12 September 2007

Online at stacks.iop.org/MST/18/3211

\begin{abstract}
The behaviour of distributed temperature sensors based on spontaneous Raman scattering and coded OTDR (optical time domain reflectometry) is studied both theoretically and experimentally; in particular a high performance scheme has been implemented using amplitude modulation according to Simplex coding, direct detection and additional use of lumped Raman amplification to further extend the sensing range. An efficient and cost-effective distributed temperature sensing system operating along $30 \mathrm{~km}$ of dispersion-shifted fibre with $17 \mathrm{~m}$ spatial resolution and $5 \mathrm{~K}$ temperature resolution is theoretically demonstrated and experimentally achieved using 255 bit Simplex coding and low-power commercially available laser diodes ( $80 \mathrm{~mW}$ CW power). Use of lumped Raman amplification to produce high-power coded pulses allows further $10 \mathrm{~km}$ distance enhancement, resulting in a total measurement range of $40 \mathrm{~km}$.
\end{abstract}

Keywords: fibre optics, sensors, Raman scattering, fibre testing, coding

\section{Introduction}

Raman-based distributed temperature sensors (DTS) have been the subject of intense research for several years [1] and, due to their wide range of practical applications, they are today available as commercial products.

The sensing principle is based on optical time domain reflectometry (OTDR) in which pump light pulses are sent down along the sensing fibre and the backward propagating light is detected. Most Raman-DTS systems based on spontaneous Raman scattering (SRS) light monitor the ratio of the temperature-dependent anti-Stokes to the Stokes light intensities, thus allowing for a correction of most fibre link losses. However, temperature assessment can also be based on the ratio of the anti-Stokes to the Rayleigh backscattered light intensities.

Being based on well-understood and mature technology principles, the range of applications of Raman-based DTS is to-date rapidly growing, mostly including monitoring of oil/gas pipelines and power cables, fire detection systems in tunnels and nuclear plants [2, 3], as well as aeronautic applications [4].

Most Raman-based DTS commercial systems are based on multimode fibre technology and are targeting sensing distances within the range of $2-10 \mathrm{~km}$.

For longer sensing distances, the development and commercial exploitation of low-cost DTS systems is however hindered by the low backscattered intensity of the anti-Stokes light, which requires high peak power levels in the OTDR, as well as high sensitivity detection schemes, in order to make the sensor performance attractive. These features have made other DTS systems more attractive and cost effective for long distance applications, such as spontaneous Brillouin scattering-based sensors [5].

We have recently shown that coded OTDR techniques with fibre link optimization allow for enhanced performance 
in Raman-based DTS, avoiding the use of high peak power pump pulses [6].

In particular, using 255 bit Simplex codes and low-power commercially available laser diodes at $1.55 \mu \mathrm{m}$, we have demonstrated a DTS system based on a dispersion-shifted (DS) fibre with $17 \mathrm{~m} / 3 \mathrm{~K}$ spatial/temperature resolution and with a measurement range enhancement greater than $15 \mathrm{~km}$ with respect to conventional OTDR-based DTS systems operating on conventional single mode fibre. Note that this has been achieved using off-shell conventional OTDR hardware and standard third window optical communication components, providing then a cost-effective solution with a maximum sensing distance up to more than $30 \mathrm{~km}$, using dispersion-shifted fibre.

A possible way to further enhance the measurement range without sacrificing spatial and temperature resolution, consists in introducing optical amplification techniques.

Distributed Raman amplification, although very effective in improving the performance of Brillouin-based DTS, does not seem to be appropriate in the case of Raman DTS; this is due to the wide spectral band of the used pump laser at $1.55 \mu \mathrm{m}$, which avoids the use of coherent detection, but unfortunately does not allow one to effectively filter out the amplified spontaneous emission added along the sensing fibre by the distributed amplification process. However, discrete (or lumped) optical amplifiers can be used to enhance the peak power of the coded OTDR pulses.

In this paper, using lumped optical amplification in combination with 255 bit Simplex coded pulses along zerodispersion-shifted sensing fibres, we demonstrate a sensing range enhancement up to $10 \mathrm{~km}$ by amplifying the coded OTDR signal at $1.55 \mu \mathrm{m}$ before launching it into the sensing fibre; a measurement distance greater than $40 \mathrm{~km}$ is reported, with spatial (temperature) resolution of $17 \mathrm{~m}(5 \mathrm{~K})$.

Since standard erbium-doped fibre amplifiers and counterpumped discrete Raman amplifiers cannot be effectively used for coded OTDR amplification (due to undesirable waveform distortion related to slow transient effects), we have developed a co-pumped low-noise discrete-or lumpedRaman amplifier based on the DS fibre, which provides up to $6 \mathrm{~dB}$ net gain to the coded OTDR signal. Standard fibre Raman laser (FRL) technology at $1460 \mathrm{~nm}$ is used to co-pump the discrete Raman amplifier; no penalties associated with pump to OTDR signal noise transfer have been observed, due to the limited bandwidth of the receiver.

The paper is organized as follows: we first introduce the basic theory of Raman-based distributed temperature sensors and then introduce the experimental set-up including opticalcoded OTDR and discrete Raman amplification. Analysis of lumped amplification is then carried out and compared with the most common optical amplification techniques. Experimental results are hence presented, showing the obtained enhancement in the measurement distance. Also theoretical calculations have been carried out, well predicting the S-coding gain as well as the performance enhancement due to discrete optical amplification of the coded OTDR pulses.

\section{Theory}

In OTDR-based DTS systems, short laser pulses are sent along the sensing fibre, and the backscattered light, containing information about loss and temperature along the fibre, is detected with high temporal resolution. In particular, as the anti-Stokes line depends on both fibre temperature (due to changes in phonon distribution) and fibre loss, the ratios of anti-Stokes to Stokes light intensities or anti-Stokes light (AS) to backscattered pump light (BS) intensities are typically used for effectively distinguishing distributed temperature variations from local losses.

In our measurements we use the ratio between the backward propagating AS intensity $I_{\mathrm{AS}}$ and the BS pump intensity $I_{\mathrm{BS}}$, which can be approximately expressed (at the scattering point) as

$$
\frac{I_{\mathrm{AS}}}{I_{\mathrm{BS}}} \propto\left[\exp \left(\frac{h \Delta v_{R}}{k T}\right)-1\right]^{-1},
$$

where $h$ is the Planck constant, $k$ is the Boltzmann constant, $T$ is the absolute temperature, and $\Delta v_{R}$ is the separation between Raman anti-Stokes and pump light frequencies.

Equation (1) must be properly integrated along the distance and spectrum to take into account the wavelengthdependent loss along the light path and the cross-section spectrum of Raman anti-Stokes scattering.

In more detail, the amount of optical power incident at the receiver deriving from pump Rayleigh backscattering and antiStokes light scattering at distance $z$ can be written respectively as

$$
P_{\mathrm{BS}}(z)=T_{\mathrm{BS}} \frac{W}{2} R_{\mathrm{BS}}(z) P_{0} \exp \left[-2 \int_{0}^{z} \alpha_{\mathrm{BS}}(\varsigma) \mathrm{d} \varsigma\right]
$$

and

$$
\begin{aligned}
& P_{\mathrm{AS}}(z)=T_{\mathrm{AS}} \frac{W}{2} R_{\mathrm{AS}}(z) P_{0} \\
& \quad \times \exp \left\{-\int_{0}^{z}\left[\alpha_{\mathrm{BS}}(\varsigma)+\alpha_{\mathrm{AS}}(\varsigma)\right] \mathrm{d} \varsigma\right\},
\end{aligned}
$$

where $W$ is the pulse width, $P_{0}$ is the input pump peak power, $\alpha_{\mathrm{BS}}$ and $\alpha_{\mathrm{AS}}$ are the loss coefficients at BS and AS light wavelengths, respectively. $T_{\mathrm{BS}}$ and $T_{\mathrm{AS}}$ are the transmittivity values at the receiver end for AS and BS light, respectively. $R_{\mathrm{BS}}(z)$ is the Rayleigh backscattering coefficient at position $z$, and can be written as

$$
R_{\mathrm{BS}}(z)=S(z) \cdot \alpha_{S}(z),
$$

where $S(z)$ is the fibre capture factor at $z$ (depending essentially on the fibre type) and $\alpha_{s}$ is the fraction of absorption coefficient due to Rayleigh scattering $\left(\propto 1 / \lambda^{4}\right)$ for BS light.

$R_{\mathrm{AS}}(z)$ is the temperature-dependent Raman backscattering coefficient [7], and its expression can be written as

$$
\begin{aligned}
& R_{\mathrm{AS}}(z)=C \cdot g_{R}(z) \int_{v_{p} \in S_{p}} S_{p}\left(v_{p}\right) \mathrm{d} v_{p} \\
& =\int_{v_{1}-v_{p}}^{v_{2}-v_{p}} \sigma_{R}\left(v_{R}\right) B\left(v_{R}+v_{p}\right)\left\{\exp \left[\frac{h v_{R}}{k_{B} T(z)}\right]-1\right\} \mathrm{d} v_{R}
\end{aligned}
$$

where $g_{R}(z)$ is the fibre Raman gain efficiency, $S_{p}\left(v_{p}\right)$ is the pump optical power spectrum, $\sigma_{R}\left(v_{R}\right)$ is the normalized crosssection of Raman scattering, $B(v)$ is the spectral band-pass filter shape, $C$ is a constant proportionality factor, $h$ and $k_{B}$ are the Planck and Boltzmann constants, $T(z)$ is the fibre temperature at position $z$. Double integration is performed to take into account spectral widths of pump light and Raman 


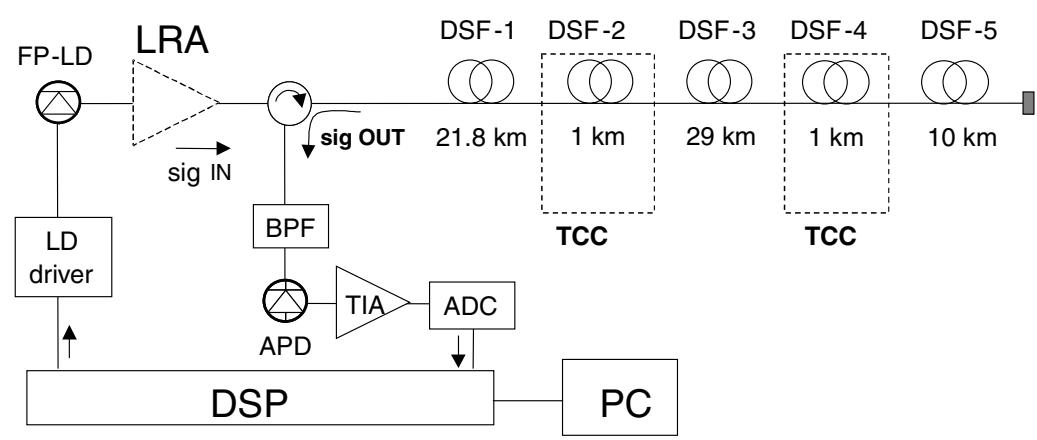

Figure 1. Experimental set-up implementing Raman-based DTS with coded OTDR.

scattering effect. In addition, equation (5) accounts for the non-ideal spectral response of the band-pass filter used to separate the AS light from BS light.

In order to compensate for possible changes in fibre loss due to connectors, bends and so on, temperature distribution is derived by using the ratio of $P_{\mathrm{BS}}(z)$ to $P_{\mathrm{AS}}(z)$, giving

$$
\frac{P_{\mathrm{BS}}(z)}{P_{\mathrm{AS}}(z)}=\frac{T_{\mathrm{BS}}}{T_{\mathrm{AS}}} \frac{R_{\mathrm{BS}}(z)}{R_{\mathrm{AS}}(z)} \exp \left\{-\int_{0}^{z}\left[\alpha_{\mathrm{BS}}(\varsigma)-\alpha_{\mathrm{AS}}(\varsigma)\right] \mathrm{d} \varsigma\right\} \text {. }
$$

Using equation (6) it is difficult to estimate the absolute fibre temperature, but it is possible to accurately measure the temperature variations along the whole optical fibre with respect to the known absolute temperature ( $300 \mathrm{~K}$ in our case) at the fibre input.

Coding techniques in OTDR schemes provide substantial SNR improvements, which can be quantified by the coding gain, defined as the ratio of SNR obtained with coded OTDR to SNR obtained with conventional ODTR at a given distance, and equal total number of measured traces.

Regarding the Simplex coding scheme, used in this work, the coding process is implemented by amplitude modulating a laser diode with sequences of pulses, according to code words which are row vectors of an unipolar $S$-matrix [8]. Decoding is achieved by a simple linear algebra operation on the set of measured coded OTDR traces obtained from the different code words. This provides an effective noise reduction in the decoded trace, with respect to the single-pulse case, and a consequent increase in the signal-to-noise ratio with respect to conventional, single-pulse OTDR, for the same number of acquired traces. The signal-to-noise ratio enhancement provided by coding is quantified by the coding gain $G_{\text {cod }}$, which, for an arbitrary code length $L$ and under the assumption of large receiver bandwidth and zero-mean uncorrelated noise, is given by

$$
G_{\mathrm{COD}}=\frac{L+1}{2 \sqrt{L}} .
$$

In a more realistic case, considering a narrow receiver bandwidth $B$, and assuming ideal low-pass response [8], the coding gain can be written as

$$
G_{\mathrm{COD}}=\frac{L+1}{2 \sqrt{L}}\left[\sqrt{1-\frac{1}{L^{2}} \sum_{n=1}^{L}(L-n) \frac{\sin (2 n \pi B \tau)}{2 n \pi B \tau}}\right]^{-1}
$$

where $\tau$ is the pulse period. As can be readily verified, for our used bandwidth (3 MHz) and pulse duration conditions (100 ns), the two equations give negligible differences in expected coding gain. However, for narrower receiver bandwidths, significant further improvement in coding gain can be achieved (at the expense of worse spatial resolution). It is also worth noting that Simplex coding can provide SNR enhancement without sacrificing spatial resolution unlike correlation-based coding techniques which degrade spatial resolution performance to some extent [8].

Finally, note that the longer coded pulse duration for 255 bit code words $(25 \mu \mathrm{s})$ does not decrease the power threshold for the onset of stimulated Raman scattering, since for pulses longer than about $100 \mathrm{~ns}$ the threshold power is nearly constant $(\sim 1 \mathrm{~W})$ and does not depend on the pulse width $[9,10]$.

\section{Experiment}

Figure 1 shows the experimental set-up used for implementing the Raman-based DTS system based on coded OTDR and lumped Raman amplification of OTDR coded pulses. The laser diode at $1550 \mathrm{~nm}$ was intensity modulated (direct current modulation through a laser driver module) by an in-house-built PC-controlled OTDR board with a digital signal processor (DSP), able to generate pulse patterns of Simplex coding [6], with $100 \mathrm{~ns}$ single bit pulse width, as well as to implement single pulses as required in conventional OTDR.

The light source was a commercially available FabryPerot laser diode (FP-LD centred at $1550 \mathrm{~nm}, 80 \mathrm{~mW}$ maximum output power, $10 \mathrm{~nm}$ FWHM, thus preventing coherent speckles). The input pulses were injected into the sensing fibre through an optical circulator, and the backscattered light wave signals were then coupled to the receiver after a large-bandwidth band-pass filter (bandwidths are about $1420-1510 \mathrm{~nm}$ for anti-Stokes light and 1520 $1600 \mathrm{~nm}$ for Rayleigh-scattered pump light). Measured transmittance spectra of BPF for the anti-Stokes and Stokes light are reported in figure 2 , showing a directivity higher than $\sim 30 \mathrm{~dB}$ for Rayleigh-backscattering port, and higher than $\sim 43 \mathrm{~dB}$ for anti-Stokes port, which permits the effective separation of anti-Stokes and Rayleigh-scattering light.

In this paper we compare the performance of standard OTDR, 255 bit S-coded OTDR and 255 bit S-coded OTDR combined with a lumped Raman amplification scheme (LRA), whose structure is schematically shown in figure 3 , to provide 


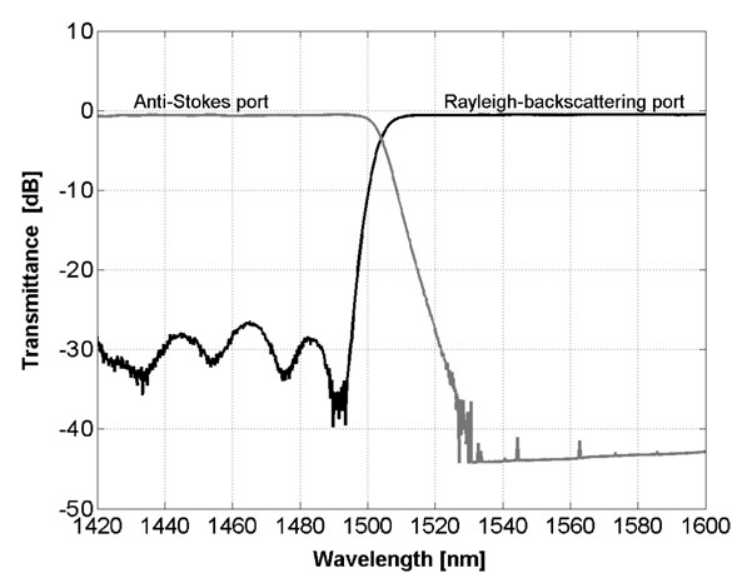

Figure 2. Transmittance spectra at BPF output.

low-noise optical amplification for the long coded pulse pattern at $1550 \mathrm{~nm}$, as explained below.

The receiver, described in figure 1 , is based on a high sensitivity InGaAs avalanche photodiode (APD), amplified by a high gain transimpedance amplifier (TIA), with about $3 \mathrm{MHz}$ bandwidth. The estimated temperature sensitivity with this set-up was about $0.65 \% / \mathrm{K}$ (at $T=300 \mathrm{~K}$ ).

After the receiver block, an analogue-to-digital converter (ADC) was used to sample the incoming analogue data trace at $20 \mathrm{MHz}$. The OTDR traces resulting from coded pulses were hence transmitted to the PC, where the de-coding process was carried out. The spatial resolution (defined by the measured $10 \%-90 \%$ response time) for the current DTS system was found to be $17 \mathrm{~m}$, mainly limited by the TIA bandwidth.

Five spools of single-mode dispersion-shifted fibres (DSF-1 to DSF-5) have been used (lengths are 21.8, 1, 29, 1 and $10 \mathrm{~km}$, respectively). The fibres have been spliced together to compose a total of $62.8 \mathrm{~km}$ sensing fibre link. DSF-2 and DSF-4 have been put inside a temperaturecontrolled chamber (TCC), while the other spools were kept at room temperature $(300 \mathrm{~K})$. DSF are characterized by smaller chromatic dispersion and higher Raman scattering coefficient than standard SMF (showing similar loss coefficients), and hence they have been found more suitable for long-range Raman-based DTS [6].

\section{Optical-coded pulse amplification}

In order to increase the peak power at the DTS input, thus permitting enhancement of the sensing distance in a Raman- based DTS, the coded sequences at the output of the FP laser have been optically amplified. The long used code words, and the high required values of optical peak power impose serious constraints for a suitable amplification technique, as explained below. The proposed scheme is shown in figure 3, and is based on a LRA. In this scheme, a WDM combiner couples in the co-propagating direction the coded pulses from directly modulated FP-LD $\left(1550 \mathrm{~nm}, P_{\text {peak }}=80 \mathrm{~mW}\right)$ together with a non-polarized FRL pump $\left(1460 \mathrm{~nm}, P_{\max }=1.9 \mathrm{~W}\right)$, into a DSF spool $\left(4.7 \mathrm{~km}, \lambda_{0}=1540 \mathrm{~nm}\right)$. At the LRA output, two cascaded WDM combiners have been used to separate the amplified OTDR pulses from the residual pump power with high directivity, to avoid leakage of strong FRL residual light into the sensing fibre.

The LRA stage provides a net gain of about $6 \mathrm{~dB}$, allowing one to attain about $320 \mathrm{~mW}$ peak power in coded pulse patterns, with negligible power excursions within one codeword (note that, as mentioned above, peak power remains below the threshold value for the onset of detrimental nonlinear effects $[9,10]$, thus allowing for spontaneous Raman temperature sensing).

Note that the LRA shown in figure 3 is based on a copumping scheme; actually, the use of other amplification techniques, such as counter-pumped LRA or erbium-doped fibre amplifiers (EDFAs) would not be effective due to undesirable waveform distortions of the coded OTDR signal at $1550 \mathrm{~nm}$, induced by power transient excursions [11] arising due to long codeword duration (about $25 \mu \mathrm{s}$ for a 255 bit codeword). Transient power variation within one codeword would cause significant impairment during the decoding process, leading thus to the impossibility of reconstructing the OTDR trace accurately. Semiconductor optical amplifiers (SOAs) also do not appear suitable for coded pulse amplification, due to typical lower values of saturation output power.

In order to characterize the extent of transient effects for the above-mentioned amplification techniques, a numerical study has been carried out simulating the power waveform at amplifier output in the case of 255 bit long coded sequences, considering a high output power EDFA, a counter-propagating LRA, and finally the co-propagating LRA previously described. The numerical method used to assess the transient dynamics of EDFAs is based on an average inversion model [12], also called reservoir model, where the upper level population of erbium ions and the exponential gain coefficient are averaged along the fibre length, thus describing the time-dependent gain of EDFA with a single ordinary differential equation for the average inversion level, which

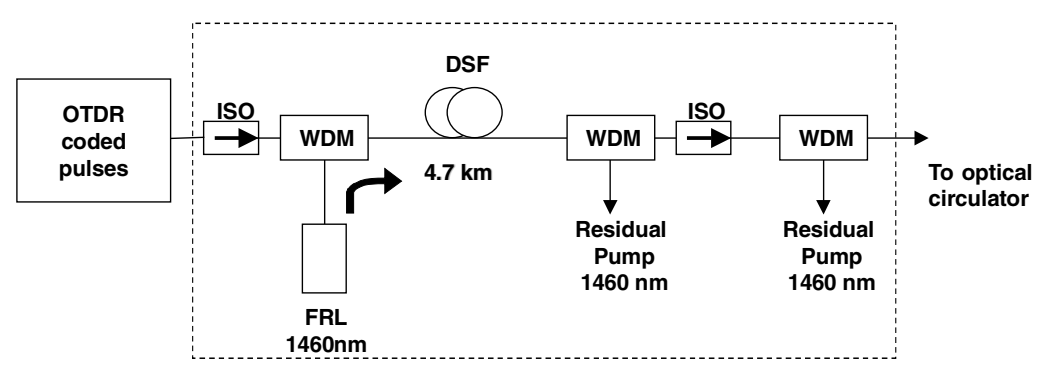

Figure 3. Scheme of co-pumped lumped Raman amplifier used to amplify long coded pulse sequences. 
can be expressed as

$$
\left(\frac{\mathrm{d}}{\mathrm{d} t}+\frac{1}{\tau_{0}}\right) \bar{N}_{2}(t)=-\frac{1}{\tau_{0} l \zeta} \sum_{i=1}^{N} P_{i}^{\mathrm{in}}(t)\left\{\exp \left[\bar{g}_{i}(t) l\right]-1\right\}
$$

where $\bar{N}_{2}(t)$ is the averaged fraction of erbium ions in the upper level at time $t, P_{i}^{\text {in }}(t)$ is the $i$ th channel input power $(N$ channels total), $l$ is the fibre length, $\tau_{0}$ is the erbium ion level lifetime, $\zeta$ is the saturation parameter or the spontaneous decay rate per unit length [12]. $\bar{g}_{i}(t)$ is the averaged gain coefficient of the $i$ th channel, expressed as $\bar{g}_{i}(t)=\left(\gamma_{i}+\alpha_{i}\right) \bar{N}_{2}(t)-\alpha_{i}$, where $\gamma_{i}$ and $\alpha_{i}$ are emission and absorption constants for the $i$ th channel. Once $\bar{N}_{2}(t)$ is calculated by integration of the ordinary differential equation system in equation (9) (and $\bar{g}_{i}(t)$ is determined from the above expression), then the output power $P_{i}^{\text {out }}(t)$ is obtained from

$$
P_{i}^{\text {out }}(t)=P_{i}^{\text {in }}(t) \exp \left[\bar{g}_{i}(t) l\right] .
$$

The tools describing dynamics in co- and counter-propagating LRAs are based on integration of a system of coupled partial differential equations in the time and space domain [11]; such a system can be generally written, with compact notation for the $i$ th signal and pump, as

$$
\begin{aligned}
& \left(\frac{\partial}{\partial z} \pm \frac{1}{V_{S, P}} \cdot \frac{\partial}{\partial t}\right) P_{S_{i}, P_{i}}^{ \pm}(z, t) \\
& =\mp \alpha_{S_{i}, P_{i}} P_{S_{i}, P_{i}}^{ \pm}(z, t) \pm \gamma_{i} P_{S_{i}, P_{i}}^{\mp} \\
& \quad \pm \sum_{j \neq i} C_{i j} P_{S_{i}, P_{i}}^{ \pm} \cdot\left[P_{p_{j}}^{+}+P_{p_{j}}^{-}\right] \\
& \quad \pm \sum_{j \neq i} C_{i j} P_{S_{i}, P_{i}}^{ \pm} \cdot\left[P_{S_{j}}^{+}+P_{S_{j}}^{-}\right],
\end{aligned}
$$

where $P_{S, P}$ represent the signal and pump power, the superscript \pm represents the co- or counter-propagating direction, $V_{S, P}$ and $\alpha_{S, P}$ are the group velocity and absorption coefficient for signals/pumps, $\gamma_{i}$ is the Rayleigh scattering coefficient and $C_{i j}$ is the Raman gain efficiency [11]. Such a system is solved with the standard fourth-order Runge-Kutta method and appropriate initial conditions for input signal waveform to provide for evolution of signal power at LRA output.

The simulated 255 bit long coded sequences have $100 \mathrm{~ns}$ single pulse duration, and the amplification stage is designed in order to provide $6 \mathrm{~dB}$ net gain (including insertion loss of WDM coupler and isolators), reproducing the experimental conditions.

The simulated waveform of 255 bit coded pulse at the EDFA output can be seen in figure $4(a)$. Because of the slow transient effect of EDFA, power variation as much as $200 \mathrm{~mW}$ (more than $3 \mathrm{~dB}$ variation) occurs when the used pump power and fibre length are $200 \mathrm{~mW}$ at $980 \mathrm{~nm}$ and $1.2 \mathrm{~m}$, respectively. This variation makes impossible the decoding process described above, which assumes constant launched optical peak power during the whole measurement process.

The results of simulations of counter-propagating LRA behaviour for a single 255 bit Simplex codeword are reported in figure $4(b)$. The simulated scheme consists of a LRA whose structure is similar to the one shown in figure 3, where the pump $(1460 \mathrm{~nm})$ is now coupled at the end of DSF spool in the

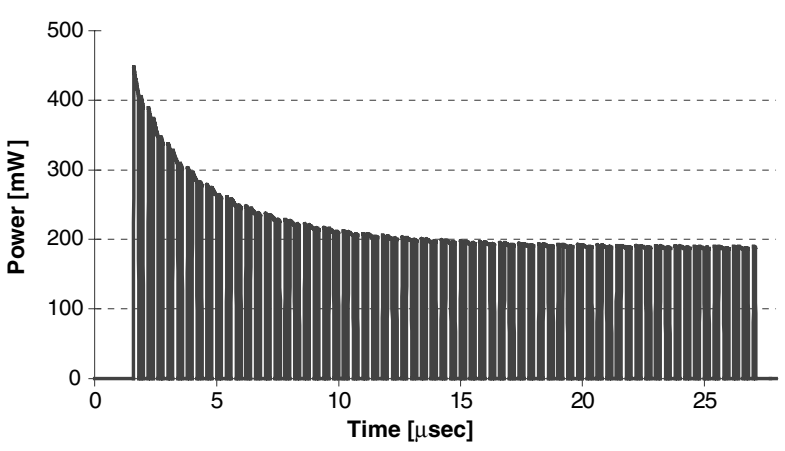

(a)

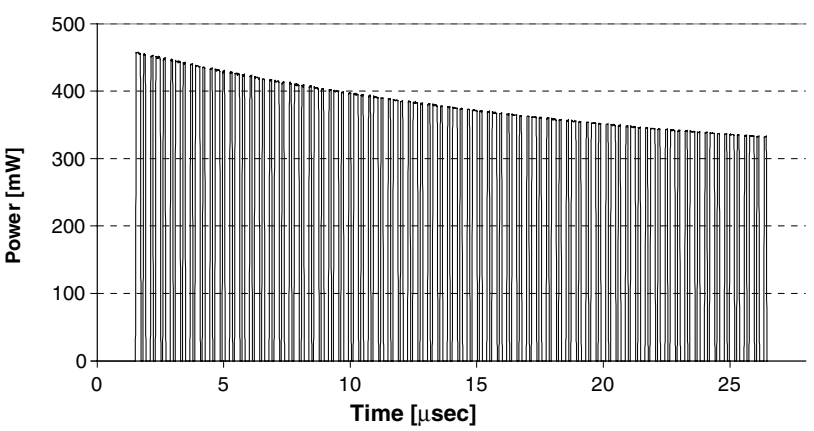

(b)

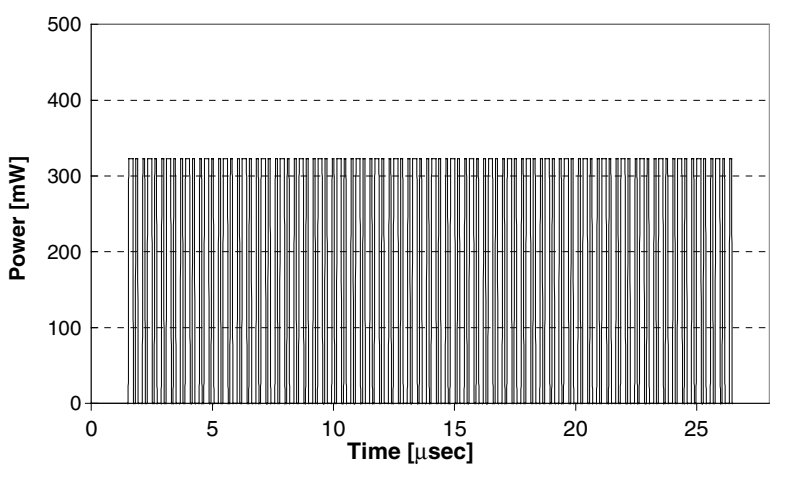

(c)

Figure 4. Output waveform for a single 255 bit codeword using: (a) an EDFA, (b) a counter-pumped LRA and (c) a co-pumped LRA.

counter-propagating direction with respect to the modulated $1550 \mathrm{~nm}$ light. An input pump power of about $1.1 \mathrm{~W}$ into the DS fibre allows for the required net gain value $(6 \mathrm{~dB}$ as in experiment).

It is evident from figure 4(b) that the counter-propagating LRA induces transient power variation within a single codeword, with a timescale of approximately $14 \mu \mathrm{s}(1 \mathrm{~dB}$ variation). The maximum power excursion for one 255 bit codeword is found to be $123 \mathrm{~mW}(1.5 \mathrm{~dB}$ relative variation), thus causing serious problems in the decoding process described above.

These impairments can be avoided by employing a copumped LRA scheme (reported in figure 3 ). Simulation results for one 255 bit codeword are presented in figure 4(c); it is evident how the faster response time characterizing co-pumped LRAs allows for a constant output peak power in amplified long coded sequences. Pump power at the DS fibre input is $1.2 \mathrm{~W}$, which is in agreement with the experimental data. 

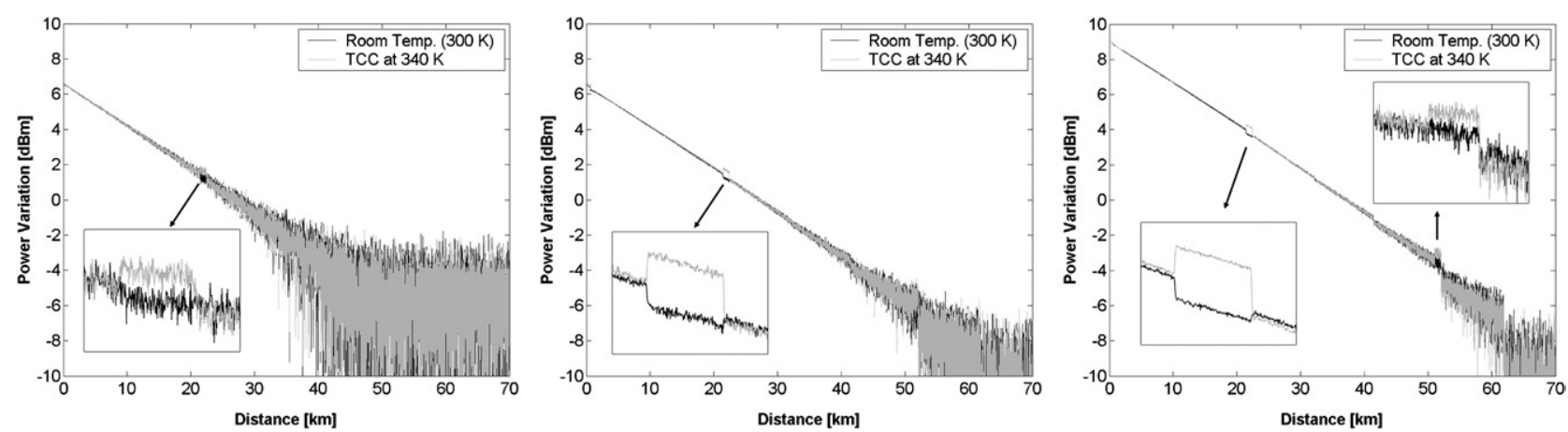

Figure 5. Traces of received anti-Stokes light power obtained using ( $a$ ) conventional OTDR, $(b)$ coded OTDR (255 bit Simplex coding), and (c) coded OTDR and lumped Raman amplification.

Note that the use of the DS fibre in LRA is crucial for obtaining optical amplification with negligible power excursion within one codeword; in fact the low chromatic dispersion value $\left(D_{M}\right)$ midway between pump and signal wavelengths results in a small walk-off during amplification with respect to bit duration, and negligible transient effects due to cross-gain saturation from neighbouring bits. More specifically, the response timescale of the co-pumped LRA can be inferred, as an order of magnitude, by estimating the walk-off parameter $W$ between pump and signal lights. This can be written in the co-pumping case as

$$
W=D_{M} \cdot \Delta \lambda \cdot L
$$

where $\Delta \lambda$ is the wavelength separation between pump at $1460 \mathrm{~nm}$ and $1550 \mathrm{~nm}$ light, and $L$ is the fibre length. In our set-up, total walk-off at the LRA output has been calculated to be about $W=1.5 \mathrm{~ns}$, that is a negligible fraction of one bit duration (100 ns), accounting for the negligible observed transient effects shown in figure 4(c).

Moreover, the high relative intensity noise value of standard FRL technology is not an issue in this application due to the limited receiver bandwidth (a few MHz), contrary to what happens for optical Raman amplification in high bitrate (e.g., $10 \mathrm{~Gb} \mathrm{~s}^{-1}$ ) transmission systems, where co-pumping with FRL generated strong signal degradation due to relative intensity noise transfer [13].

\section{Results}

The temperature estimation has been derived from the OTDR trace measurements on the sensing fibre spools using conventional pulsed OTDR, coded OTDR and coded OTDR in combination with lumped Raman amplification. Two different temperature conditions have been considered in all cases: in the first one, all fibre spools are kept at room temperature $(300 \mathrm{~K})$, in the second condition, two fibre spools (DSF-2 and DSF-4) have been heated up to $340 \mathrm{~K}$ in a temperaturecontrolled chamber (see figure 1).

Figure 5 reports the obtained OTDR traces for anti-Stokes light with all sensing fibre spools kept at room temperature (black line), and with the two spools (DSF-2 and DSF-4) heated at $340 \mathrm{~K}$ (grey line), showing the expected increase in anti-Stokes intensity for the heated fibre spools. Figure 5(a) reports the curves obtained with conventional OTDR and 180000 time averages, figure 5(b) shows the traces obtained

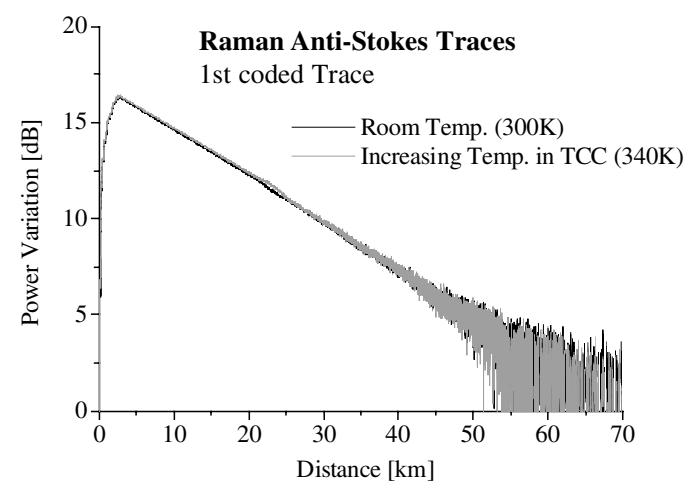

Figure 6. Acquired trace of anti-Stokes light power for the first codeword of 255 bit Simplex-coded OTDR.

using 255 bit S-coded OTDR (80 $\mathrm{mW}$ peak optical input power), and figure $5(c)$ the same traces in the case of 255 bit S-coded OTDR and additional discrete Raman amplification of the OTDR signal $(320 \mathrm{~mW}$ peak power at sensing fibre input).

Note that for a sensible comparison of standard and coded OTDR techniques, a number of 706 time averages have been used for a single codeword (255 orthogonal Simplex code words are used), thus giving the same total number of 180000 acquired traces as with conventional OTDR. An example of time-averaged trace for a single codeword is shown in figure 6, where the higher power value and the larger spatial resolution than in conventional OTDR can be noted. By conveniently combining all traces from different code words during the decoding process [8], the spatial resolution of decoded trace is then equal to the single pulse OTDR trace resolution $(17 \mathrm{~m}$ in this case).

This can be seen in figure 5, where the coded OTDR allows for a sensible increase in SNR with respect to un-coded OTDR (for the same input power and same total number of acquired traces), hence allowing for an increased measurement range of temperature sensing. Also evident is how discrete Raman amplification of the coded OTDR signal allows for higher launched power and consequent improved SNR.

The OTDR trace of Rayleigh backscattered light is also used for deriving the temperature profile, see equation (6). Since the Rayleigh scattering light intensity is several orders of magnitude stronger than the anti-Stokes intensity, the former was acquired using conventional OTDR only, allowing for 


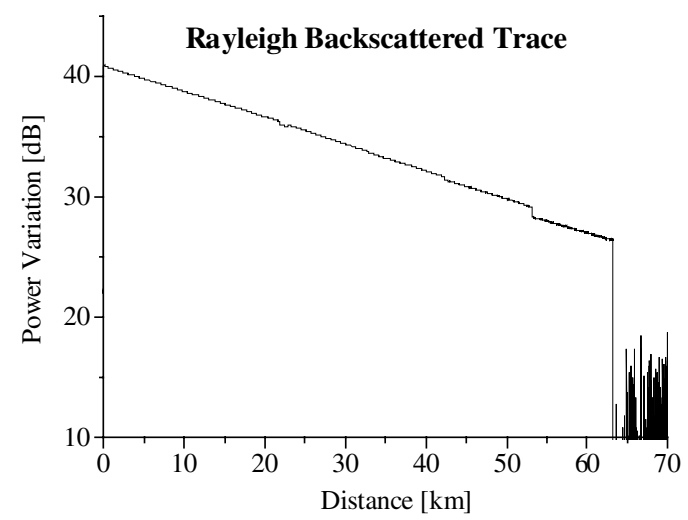

Figure 7. Trace of received Rayleigh-scattered light power obtained using conventional OTDR only.

adequate SNR values at all distances from fibre near end. The resulting trace is shown in figure 7 , where the higher power and SNR values with respect to the AS trace in figure 5(a) are clearly evident.

The ratio $P_{\mathrm{AS}} / P_{\mathrm{BS}}$, corrected for wavelength-dependent losses, has then been used for temperature assessing. The temperature distribution calculated from the anti-Stokes to Rayleigh backscattering ratio is shown in figures $8(a)-(c)$ using conventional OTDR, pulse coding and pulse coding together with LRA, respectively.

It is evident from figure 8 that the temperature distribution obtained by using 255 bit coding allows for more accurate temperature assessing and increased measurement distance at the same SNR with respect to conventional OTDR. The effect of additional discrete optical amplification is seen from figure $8(c)$; it allows for further enhancement of the sensing range with a given resolution, allowing, e.g., to detect temperature variation in DSF-4 heated fibre spool, placed at a distance of $50 \mathrm{~km}$. Achieved root-mean-square (RMS) temperature resolution versus distance is reported in figure 9, for each of the three used DTS techniques, namely, $(a)$ conventional OTDR (squares), (b) Simplex-coded OTDR (circles) and (c) Simplex-coded OTDR in association with lumped Raman amplification (triangles). For a desired temperature/spatial resolution, the measurement range is

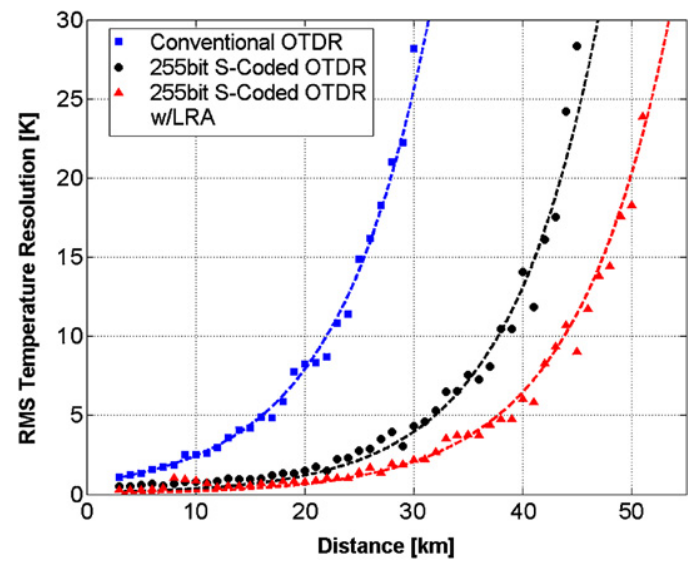

Figure 9. RMS temperature resolution versus distance (exponential fit shown in dashed lines) using conventional OTDR (squares), coded OTDR (circles) and coded OTDR with lumped Raman amplification (triangles).

(This figure is in colour only in the electronic version)

greatly enhanced by the S-coding and discrete Raman amplification. Temperature resolution versus distance is observed to have exponential behaviour, as expected from exponential SNR degradation with distance due to fibre absorption; this is shown in exponential fitting of experimental data of figure 9 (dotted lines, $\chi^{2}$-test giving $0.4,1.05$ and 1.15 for cases $(a),(b)$ and $(c)$, respectively).

In particular, by considering a reference temperature resolution of $5 \mathrm{~K}$, we can see that the sensing range is limited to less than $15 \mathrm{~km}$ with conventional OTDR, while 255 bit Simplex-coded OTDR allows for about $30 \mathrm{~km}$ measurement range (i.e., $15 \mathrm{~km}$ increase); providing discrete Raman amplification to the coded OTDR signal allows for a measurement range greater than $40 \mathrm{~km}$ (i.e., $10 \mathrm{~km}$ further increase). These experimental results are consistent with theoretical predictions; actually, based on the coding gain formula equation (8) for finite receiver bandwidth, predicted coding gain is $9 \mathrm{~dB}$ (accounting for one-way $15 \mathrm{~km}$ increase). Also the net gain provided by the LRA $(6 \mathrm{~dB})$ accounts for the $10 \mathrm{~km}$ sensing range enhancement observed when combining S-coding with discrete Raman amplification.
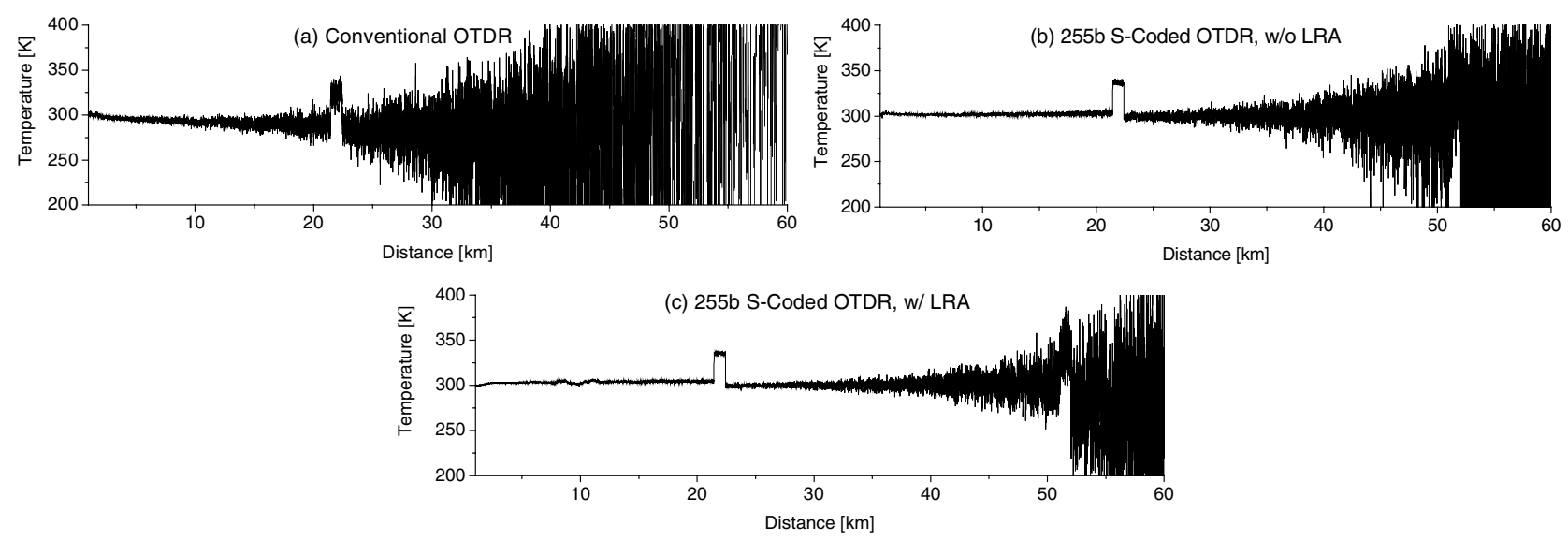

Figure 8. Temperature distribution versus fibre length obtained from measured traces using $(a)$ conventional OTDR, $(b)$ coded OTDR (255 bit Simplex codes), and (c) coded OTDR and lumped Raman amplification (DSF-2 and DSF-4 spools have been heated at 340 K). 


\section{Conclusion}

In conclusion, improved performance in Raman-based DTS systems has been demonstrated using 255 bit Simplex coded OTDR, combined with discrete Raman amplification. Experimental results show that 255 bit Simplex coded OTDR provides a sensing range enhancement, with respect to conventional OTDR, of about $15 \mathrm{~km}$ with $17 \mathrm{~m} / 5 \mathrm{~K}$ spatial/temperature resolution, making use of low-power $(80 \mathrm{~mW})$ commercially available laser diodes practical for most applications.

Further performance improvement can be achieved introducing low-noise discrete Raman amplification of coded pulses, allowing more than $40 \mathrm{~km}$ measurement range with the same spatial/temperature resolution $(17 \mathrm{~m} / 5 \mathrm{~K})$.

\section{References}

[1] Kee H H et al 19991.65 mm Raman-based distributed temperature sensor Electron. Lett. 35 1869-71

[2] Liu Z et al 2003 Review of recent developments in fire detection technologies J. Fire Prot. Eng. 13 129-51

[3] Fernandez A F et al 2005 Radiation-tolerant Raman distributed temperature monitoring system for large nuclear infrastructures IEEE Trans. Nucl. Sci. 52 2689-94
[4] Odic R M et al 2002 Distributed temperature sensor for aeronautic applications 15th Optical Fiber Sensor Conf. Technical Digest 2002, OFS 2002 (6-10 May 2002) vol 1 pp 459-62

[5] Cho Y T et al $200350 \mathrm{~km}$ single-ended spontaneous-Brillouin-based distributed-temperature sensor exploiting pulsed Raman amplification Opt. Lett. 28 1651-3

[6] Park J et al 2006 Raman-based distributed temperature sensor with Simplex coding and link optimization IEEE Photon. Technol. Lett. 18 1879-81

[7] Islam M N 2004 Raman Amplifiers for Telecommunications vol 1 (Berlin: Springer)

[8] Lee D et al 2004 Analysis and experimental demonstration of simplex coding technique for SNR enhancement of OTDR LTIMC-15: Proc. IEEE LTIMC (New York, October 2004)

[9] Wait P C et al 1997 A theoretical comparison of spontaneous Raman and Brillouin based fibre optic distributed temperature sensors Opt. Commun. 144 17-23

[10] Scheerer C 1996 OTDR pulse power limit in on-line monitoring of optical fibres owing to stimulated Raman scattering Electron. Lett. 32 679-80

[11] Bolognini G et al 2004 Transient effects in gain clamped discrete Raman amplifiers IEEE Photon. Technol. Lett. 16 66-8

[12] Sun Y et al 1997 Average inversion level, modeling, and physics of erbium-doped fiber amplifiers J. Select. Top. Quantum Electron. 3 991-1007

[13] Fludger C R S et al 2001 Pump to signal RIN transfer in Raman fiber amplifiers J. Technol. Lett. 19 1140-8 